\title{
Baicalein decreases uric acid and prevents hyperuricemic nephropathy in mice
}

\author{
Xiaolu Meng ${ }^{1}$, Zhuo Mao ${ }^{2}$, Xin $\mathrm{Li}^{3}$, Dandan Zhong ${ }^{1}$, Min $\mathrm{Li}^{1}$, Yingli Jia ${ }^{1}$, Jing $\mathrm{Wei}^{2}$, \\ Baoxue Yang ${ }^{1}$ and Hong Zhou ${ }^{1}$ \\ ${ }^{1}$ Department of Pharmacology, School of Basic Medical Sciences, Peking University, Beijing, 100191, P.R. China \\ ${ }^{2}$ Tianjin Key Laboratory for Modern Drug Delivery and High-Efficiency, School of Pharmaceutical Science and Technology, \\ Tianjin University, Tianjin, 300072, P.R. China \\ ${ }^{3}$ Drug Clinical Trial Institution, The Affiliated Hospital of Qingdao University, Qingdao, 266003, P.R. China
}

Correspondence to: Hong Zhou, email: rainbow_zhou@126.com

Keywords: baicalein, hyperuricemia, uric acid, nephropathy, xanthine oxidoreductase

Received: January 06, 2017 Accepted: March 24, $2017 \quad$ Published: April 07, 2017

Copyright: Meng et al. This is an open-access article distributed under the terms of the Creative Commons Attribution License 3.0 (CC BY 3.0), which permits unrestricted use, distribution, and reproduction in any medium, provided the original author and source are credited.

\begin{abstract}
Baicalein, a natural flavonoid, is structurally advantageous for binding to xanthine oxidoreductase. In our study, molecular docking analysis and Surface Plasmon Resonance revealed a direct interaction between baicalein and xanthine oxidoreductase. Moreover, $50 \mathrm{mg} / \mathrm{kg} / \mathrm{d}$ baicalein treatment significantly suppressed the viability of xanthine oxidoreductase in hyperuricemia mouse model. The data showed that baicalein remarkably prevented renal dysfunction, ameliorated kidney fibrosis, alleviated epithelial-mesenchymal transition and oxidative stress in hyperuricemia mice. Thus, we concluded that baicalein executed a kidney-protection action in hyperuricemia and therefore may be used as a therapeutic alternative for hyperuricemic nephropathy.
\end{abstract}

\section{INTRODUCTION}

Hyperuricemia is an independent risk factor for kidney diseases, metabolic syndrome, diabetes, hypertension and cardiovascular diseases [1-4]. Moreover, high-uric acid (UA) induces cognitive dysfunction through hippocampal inflammation in rodents and humans [5]. Also, hyperuricemia increases the 1-year mortality of STsegment elevation myocardial infarction (STEMI) patients in Killip class I [6]. Besides, the control of serum UA can prevent tumor lysis syndrome, which is a life-threatening oncologic emergency[7].

Because approximately $70 \%$ of UA is excreted from the kidney, hyperuricemia occurs when kidney function declines [8]. However, the role of elevated UA on chronic kidney diseases (CKD) remains controversial. Notably, elevated UA level induces renal dysfunction in a great deal of animal studies, but for humans, the relationship between UA and kidney disease is not that simple [9]. UA levels can be changed by other risk factors, including hypertension, metabolic syndrome and microalbuminuria, but it is not clear whether these are mediators or confounders. Most epidemiological evidence suggests a direct link between UA and CKD, but which comes first cannot be determined [10]. Therefore, there is no evidence to definitely demonstrate whether UA is causal, compensatory, coincidental or it is only an epiphenomenon in these patients [11]. At present, urate-lowering therapy in asymptomatic hyperuricemia is also conservative [12-14]. Thus, further experimental and clinical studies are still needed to identify the association between hyperuricemia and CKD.

Hyperuricemia is result from overproduction from hepatic metabolism and cell turnover or renal underexcretion. Urate is formed from dietary purines and endogenously synthesized purines. Urate is formed in the liver, where xanthine oxidoreductase (XOR) at play. XOR is a prototypical molybdenum hydroxylase, catalyzing hydroxylation of hypoxanthine to xanthine as well as xanthine to uric acid [15]. This reaction occurs at a molybdenum-pterin center. From there, the electrons are transferred via two $\mathrm{Fe}_{2} \mathrm{~S}_{2}$ clusters to the isoalloxazine ring of flavin. Then flavin passes them on to the second substrate $\mathrm{NAD}^{+}[16]$. Thus, inhibiting XOR helps downregulate UA production. As the prevalence of hyperuricemia is increasing globally, new treatment options to manage hyperuricemia is demanded. Currently, 
urate-lowering drugs contain three main classes : XOR inhibitors, uricosurics, URAT1 inhibitor, and recombinant uricases [17]. Among them, allopurinol, a XOR inhibitor, is first-line therapy [18]. However, it presents unfavorable side effects clinically, so does other medicines. So nonpurine XOR inhibitors with less toxicity grasp stronger interest. Moreover, many Chinese medical herbs are significantly superior to Western medicine in overall efficacy with fewer adverse drug reactions [19].

As a natural flavonoid extracted from the Chinese herb Scutellariae radix, baicalein has many biochemical and pharmacological benefits, including antioxidant [20], anti-inflammation [21, 22], anti-tumor [23], anti-fibrosis and cardiovascular protective effects $[24,25]$. What's more, baicalein can improve cardiac function [26], attenuate neurological deficits [27], treat gastric ulcers [28] etc. Given these data, we hypothesize that baicalein can prevent hyperuricemia and alleviate kidney damage. As baicalein exhibits strong first-pass metabolism in small intestine, we administrated baicalein 6,7-biacetate instead in our mouse model. It partly prevents glucuronidation and degrades into baicalein in intestinal track after oral administration. In our study, mouse hyperuricemia model and molecular pharmacology methods were exerted to investigate UAlowering and renal protective effects of baicalein.

\section{RESULTS}

\section{Identification of the interaction between baicalein and XOR}

Molecular docking method is used for constructing receptor-ligand complex and binding mechanism analysis. The binding modes of baicalein with rat source and human source XOR were performed and they both presented similar conformation in enzyme binding pocket (Figure 1A-D). For rat source XOR-baicalein system, Glu1261, Thr1010 of XOR binding pocket provided hydrogen bonds interaction with baicalein; Leu1011, Phe1009, Phe914, Ala1079, and Ala1078 made hydrophobic interactions with baicalein; Phe914 and Phe1009 were involved in $\pi$-cation interaction with aromatic ring of baicalein. Meanwhile, similar contributions also existed in human source XOR-baicalein system. Amino acid residues of XOR binding pocket providing hydrogen bonds included Arg881, Thr1010, and Glu1262; hydrophobic interactions were made via Phe1010, Phe915, Ala1080, Ala1079, Val808, and Val1012; Phe915 and Phe 1010 participated in $\pi$-cation interaction. The details of hydrogen bonds were shown in Tables1 and 2.

\section{The binding affinity of baicalein to XOR based on Surface Plasmon Resonance (SPR) biosensor analysis}

To verify the prediction from computational docking analysis, the binding affinity of baicalein to XOR was determined by SPR biosensor technology. Biacore 3000 instrument was utilized to record the ability of baicalein binding to XOR, which was reflected by the RU values. The RU increased as baicalein concentration was elevated, indicating that baicalein bound to XOR in a concentrationdependent manner (Figure 2A). The association $\left(\mathrm{K}_{\mathrm{on}}\right)$, dissociation $\left(\mathrm{K}_{\text {off }}\right)$, and equilibrium dissociation constants $\left(\mathrm{K}_{\mathrm{D}}\right)$ of baicalein binding to XOR were $607.7 \mathrm{M}^{-1} \mathrm{~S}^{-1}$, $9.467 \times 10^{-3} \mathrm{~S}^{-1}$, and $6.749 \times 10^{-5} \mathrm{M}$, respectively. The results showed that baicalein bound exactly to XOR.

\section{Baicalein inhibited the enzymatic activity while had no influence on the expression of XOR}

In the established hyperuricimic mouse model, the activity of XOR in liver and in serum were both elevated. Treatment with $50 \mathrm{mg} / \mathrm{kg}$ baicalein 6,7-biacetate per day effectively downregulated XOR activity both in liver and in serum (Figure 2B, 2C). However, baicalein has no significant influence on XOR expression in liver (Figure 2D).

\section{Baicalein lowered UA and protected kidney against hyperuricemia}

Renal function was assessed by serum UA(Figure 3A), urine UA (Figure 3B), UA clearance (Figure 3C), BUN (Figure 4A), serum creatinine (Figure 4B), proteinuria (Figure 4C), urine output (Figure 4D) and osmalility (Figure 4E). As shown in Figures 3 and 4, serum UA, creatinine, BUN and urinary proteinuria were all elevated in hyperuricemia mice compared with control mice, suggesting that mice develop hyperuricemic nephropathy in this model. Besides, UA clearance was significantly decreased in hyperuricemia mice (Figure 3C). Treatment with baicalein $50 \mathrm{mg} / \mathrm{kg} /$ day for 21 days improved renal function notably. As the trend of urinary osmolality was contrary to that of urine output in each group, urine concentrating ability was normal in hyperuricemia mice (Figure 4D, 4E). Furthermore, Periodic acid-Schiff staining showed that kidneys of hyperuricemia mice developed severe tubulointerstitial damage with tubular dilatation and interstitial fibrosis. In outer-medulla, we saw protein casts in dilated tubules and slight interstitial collagen accumulation. Moreover, the inner-medulla presented tubular dilatation also and the epithelial cells were disarranged. Baicalein administration preserved kidney architecture and moderated the tubulointerstitial damage (Figure 4F). Thus, baicalein can improve renal function and alleviate kidney injury in hyperuricemia.

\section{Baicalein modified XOR-dependent and NADPH oxidase-dependent renal oxidative stress in hyperuricemia mice}

XOR generates oxidative stress while the oxidative hydroxylation of xanthine to uric acid takes place. Serum 
Table 1: Distances $(\AA)$ between hydrogen bond donor and acceptor in rat source XOR-baicalein complex

\begin{tabular}{ccc}
\hline H-bond donor & H-bond acceptor & Distances $(\AA)$ \\
\hline Baicalein O4 & Glu1232 OE1 & 3.078 \\
Baicalein O4 & Glu1232 OE2 & 3.019 \\
Baicalein O5 & Glu1232 OE2 & 2.805 \\
Thr981 N & Baicalein O2 & 2.520 \\
Thr981 OG1 & Baicalein O2 & 2.647 \\
MOM OM1 & Baicalein O5 & 1.963 \\
MOM OM3 & Baicalein O5 & 3.262 \\
\hline
\end{tabular}

$\mathrm{H}_{2} \mathrm{O}_{2}$ level was elevated in hyperuricemia mice and baicalein downregulated its concentration (Figure 5A). Malondialdehyde, superoxide dismutase (SOD), reduced glutathione $(\mathrm{GSH})$ and glutathione peroxidase (GPx) were detected to evaluate the effect of baicalein on XORmediated oxidative stress in kidneys. Compared with the sham group, SOD (Figure 5B) and Mn-SOD (Figure 5C) level were suppressed while Malondialdehyde (Figure 5D) level was greatly elevated in hyperuricemia mice. GSH (Figure 5E) and GPx (Figure 5F) were also downregulated in hyperuricemia mouse. We found that 21 days of baicalein treatment at $50 \mathrm{mg} / \mathrm{kg} /$ day reversed the situation effectively. Meanwhile, we evaluate the change of NADPH oxidase 4 (Nox4), which expressed abundantly in renal

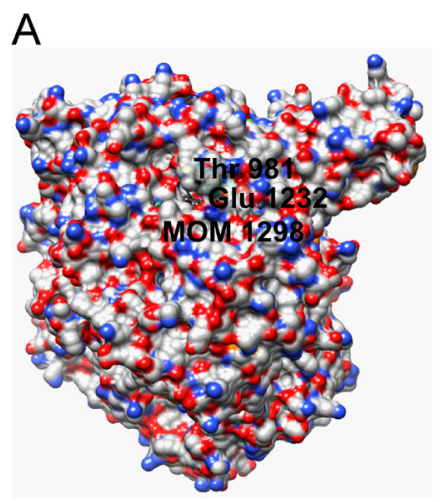

B
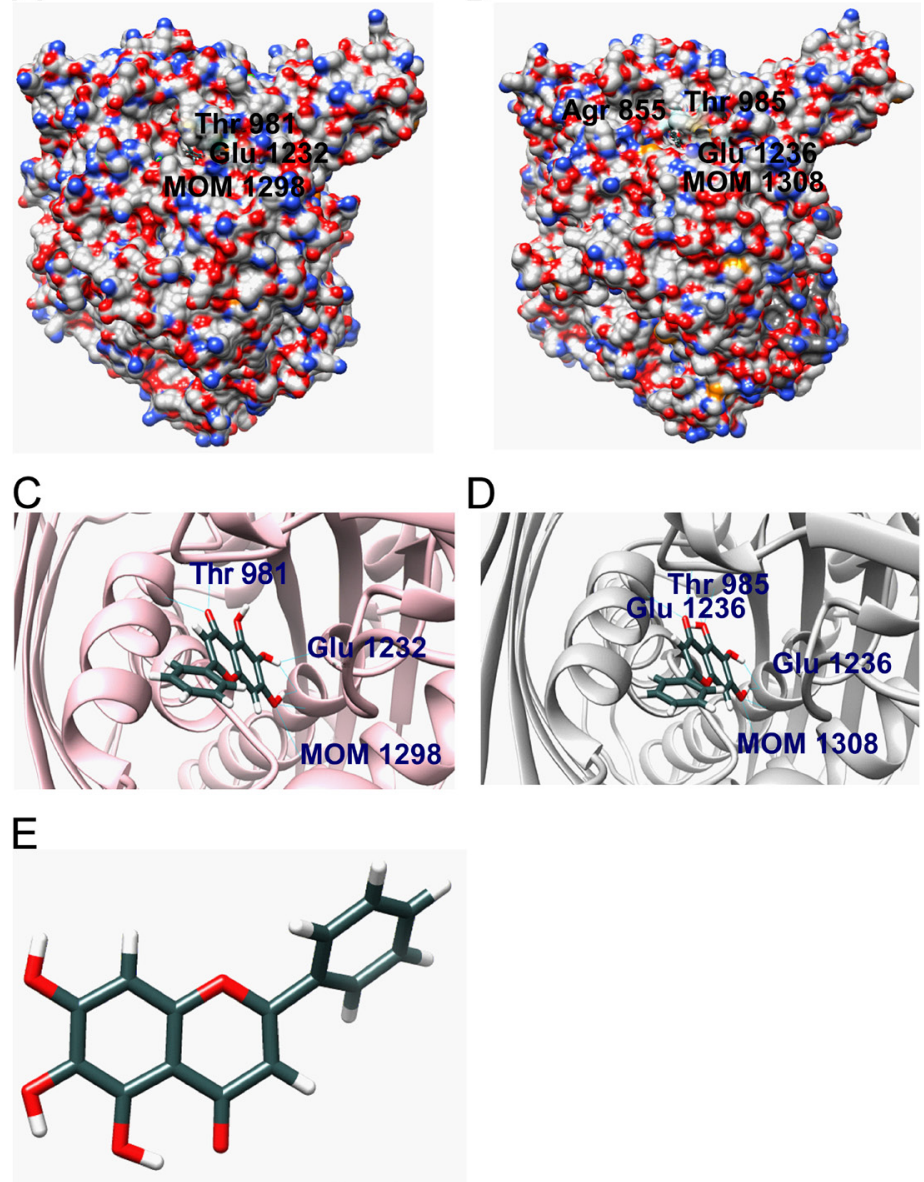

Figure 1: Molecular docking. (A) The binding model of rat source XOR-baicalein complex (XOR is shown using a surface model and baicalein is shown using a ball and stick model). (B) The binding model of human source XOR-baicalein complex (XOR is shown using a surface model and baicalein is shown using a ball and stick model). (C) The insight model of rat source XOR-baicalein complex. (D) The insight model of human source XOR-baicalein complex. (E) Molecular structure of baicalein. 
Table 2: Distances $(\AA)$ between hydrogen bond donor and acceptor in human source XORbaicalein complex

\begin{tabular}{ccc}
\hline H-bond donor & H-bond acceptor & Distances $(\AA)$ \\
\hline Baicalein O4 & Glu1236 OE1 & 3.298 \\
Baicalein O5 & Glu1236 OE2 & 2.842 \\
Thr985 N & Baicalein O2 & 2.654 \\
Thr985 OG1 & Baicalein O2 & 2.703 \\
Arg855 NE & Baicalein O3 & 2.766 \\
Arg855 NH2 & Baicalein O3 & 2.809 \\
MOM OM1 & Baicalein O5 & 1.765 \\
MOM OM3 & Baicalein O5 & 3.435 \\
\hline
\end{tabular}
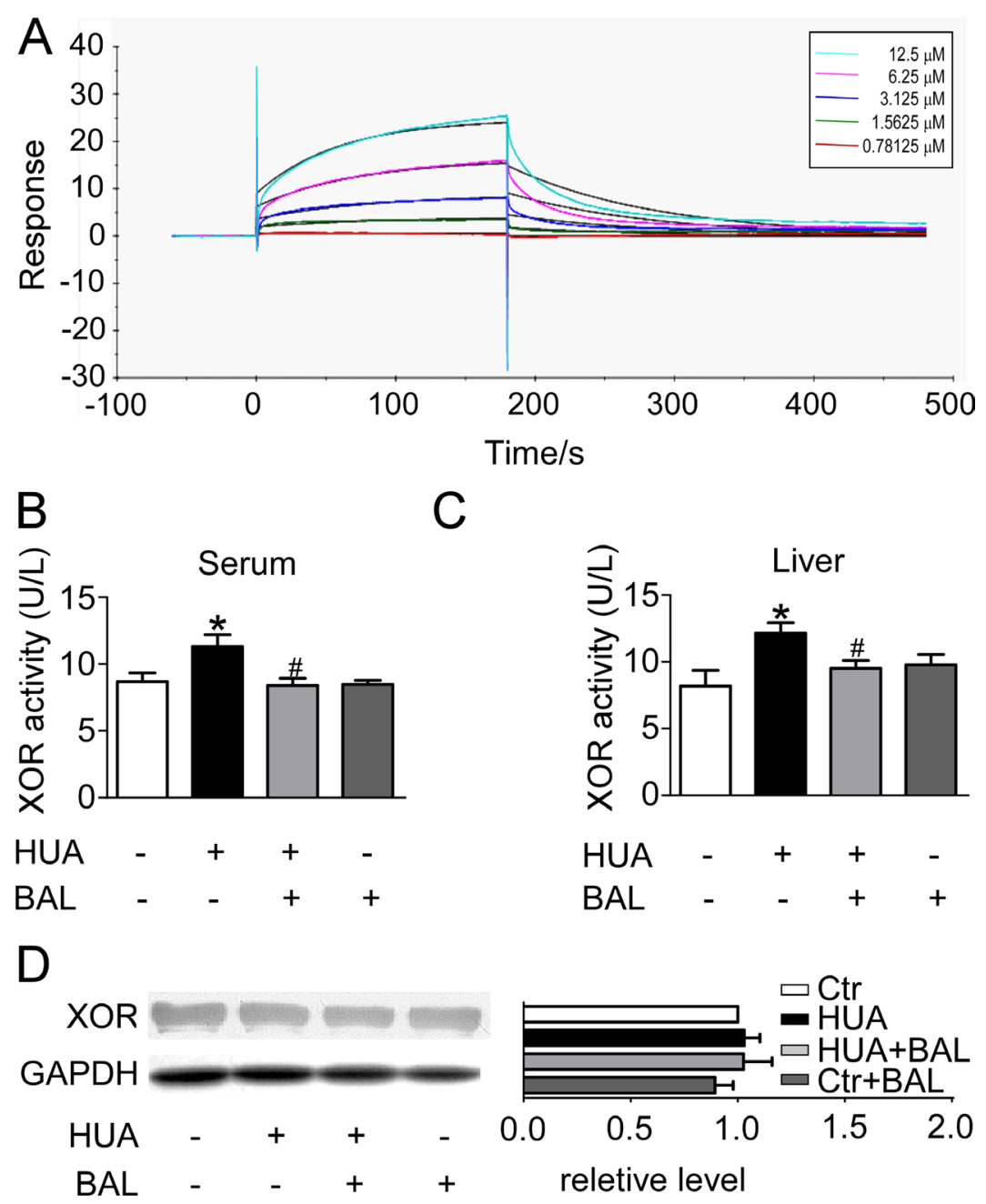

Figure 2: Binding affinity of baicalein to XOR as determined by SPR, and the inhibitory effect of baicalein to XOR in hyperuricemia mice in vivo. (A) Real-time measurements of the binding affinity of baicalein to XOR were performed using a Biacore 3000 instrument. Baicalein at a concentration of $0.78125,1.5625,3.125,6.25$ or $12.5 \mu \mathrm{M}$ (curves from bottom to top) was injected. (B) ICR male mice were intraperitoneally administered with hypoxanthine and potassium oxonate daily to establish hyperuricemia model. Baicalein was orally given to some mice. Blood samples were collected for evaluating enzymatic activity of XOR. (C) Liver tissues were homogenized for evaluating enzymatic activity of XOR. Values were means $\pm \operatorname{SEM}(n=5-6) .{ }^{*} P<0.05$ vs. control group. ${ }^{\#} P<0.05$ vs. hyperuricemia group. (D) Expression levels of XOR in liver were determined by western blots (left) and quantifications (right), which were normalized with GAPDH. Means $\pm \operatorname{SEM}(n=3)$. 
proximal tubule, and fount it upregulated in hyperuricemia mice while downregulated by baicalein (Figure 5G). These results indicated that baicalein modified XOR-dependent and NADPH oxidase-dependent renal oxidative stress in hyperuricemia mice.

\section{Baicalein suppresses hyperuricemia-induced renal fibrosis through matrix metalloproteinases (MMPs)}

Masson trichrome stain demonstrated that baicalein could moderate hyperuricemia induced renal fibrosis. Hyperuricemic mouse kidneys displayed severe morphologic lesions. It is characterized by tubular dilation with epithelial atrophy and interstitial expansion with collagen accumulation. Treatment with baicalein showed a remarkable improvement of the morphologic lesions with less fibrosis in interstitium (Figure 6A). At the same time, the expression of fibronectin, a key component of the interstitial matrix, was upregulated in hyperuricemia mice and downregulated by baicalein (Figure 6B). As MMPs were closely linked to fibrosis and changed a lot in our RNA-seq data (data not shown), we detected MMP-7 and MMP-9 level in mouse kidneys. They were downregulated and upregulated in hyperuricemia model respectively. Baicalein significantly reversed their expression (Figure 6B). Meanwhile, $\beta$-catenin level was elevated in hyperuricemia mice (Figure 6C). Therefore, baicalein may suppress hyperuricemia-induced renal fibrosis involving MMP-7 and MMP-9 signals.

\section{Baicalein inhibits hyperuricemia-induced epithelial-mesenchymal transition (EMT) process}

EMT markers were detected in our study. In hyperuricemia mouse model, the expression of E-cadherin was suppressed while $\alpha$-smooth muscle actin ( $\alpha$-SMA) was elevated remarkably, indicating EMT process. Treatment with baicalein $50 \mathrm{mg} / \mathrm{kg} /$ day blocked this pathway (Figure 6C).

\section{DISCUSSION}

In this paper, we firstly identified baicalein as an effective XOR inhibitor in vitro and in vivo. XOR is a highly versatile molybdoflavin enzyme, ubiquitous among species (from bacteria to human) and within the various tissues of mammals [15]. It catalyzes the production of UA, and is also a significant source of reactive species [29]. Thus, human XOR is a drug target for hyperuricemia treatment, and potentially for relevant diseases as well [30].

The active cavity of XOR is a long, narrow channel leading to the active site [31]. If the cavity is bound by an inhibitor, the channel and surrounding space are mostly plugged. It will hold up the landing of xanthine (substrate) and ultimately preventing its oxidation [31]. Natural products including flavonoids exhibit diversified bioactivities and lower toxicity compared with classical XOR inhibitors [32]. Flavonoids have a potent inhibitive effect towards XOR both in vitro and in vivo [33], such as
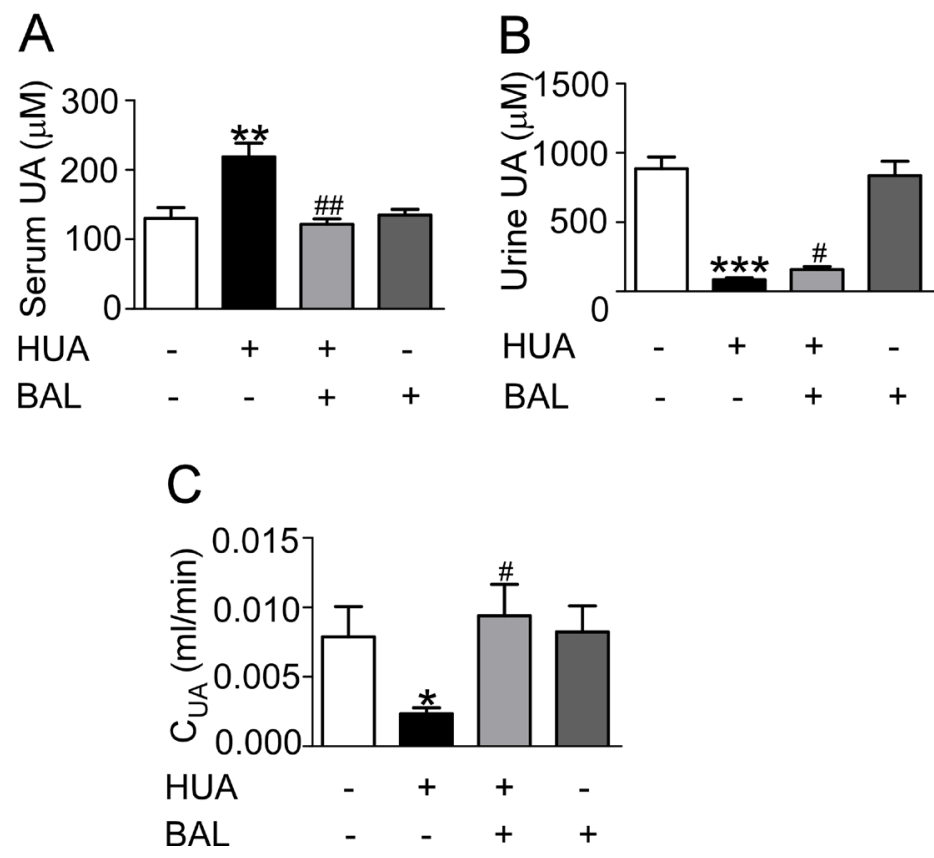

Figure 3: Baicalein reduced UA level of hyperuricemia mice in vivo. Blood and urine were collected and analyzed after hypoxanthine and potassium oxonate treatment for 2 weeks. (A) Serum UA. (B) Urine UA. (C) UA clearance. $\mathrm{C}_{\mathrm{UA}}(\mathrm{ml} / \mathrm{min})=\mathrm{urine} \mathrm{UA}$ $(\mu \mathrm{M}) \times$ urine output $(\mathrm{ml}) / \mathrm{t}(\mathrm{min}) / \operatorname{serum} \mathrm{UA}(\mu \mathrm{M})$. Values were means $\pm \mathrm{SEM}(n=5-6) . * P<0.05, * * P<0.01$ and $* * * P<0.001$ vs. control group. ${ }^{\#} P<0.05,{ }^{\#} P<0.01$ vs. hyperuricemia group. 
apigenin, luteolin, hyperin etc [34]. C2 = C3 double bonds of flavonoids contributed to their planar structure and the hydroxyl groups on C-5 and C-7 were advantageous for binding to XOR [34]. Baicalein is a natural flavonoid with all these structure superiorities above. Thus, the possibility that baicalein can inhibit XOR in vivo and fight against hyperuricemia cannot be excluded. Computational docking analysis indicated that baicalein was located in the binding pocket and interacted with several amino acid residues so as to plug the enzyme cavity. To verify the interaction between baicalein and XOR, we further evaluated their binding affinity using SPR biosensor analysis. The results confirmed the computer modeling prediction. In terms of mice and human, XOR level was highest in liver and intestine. Besides, a wide range of activity levels were in biological fluids, such as blood [35]. So we tested XOR level of liver and serum in hyperuricemia mouse model in vivo. XOR viability in liver and serum both elevated, but baicalein significantly suppressed the viability of XOR. Interestingly, the expression of XOR in liver was
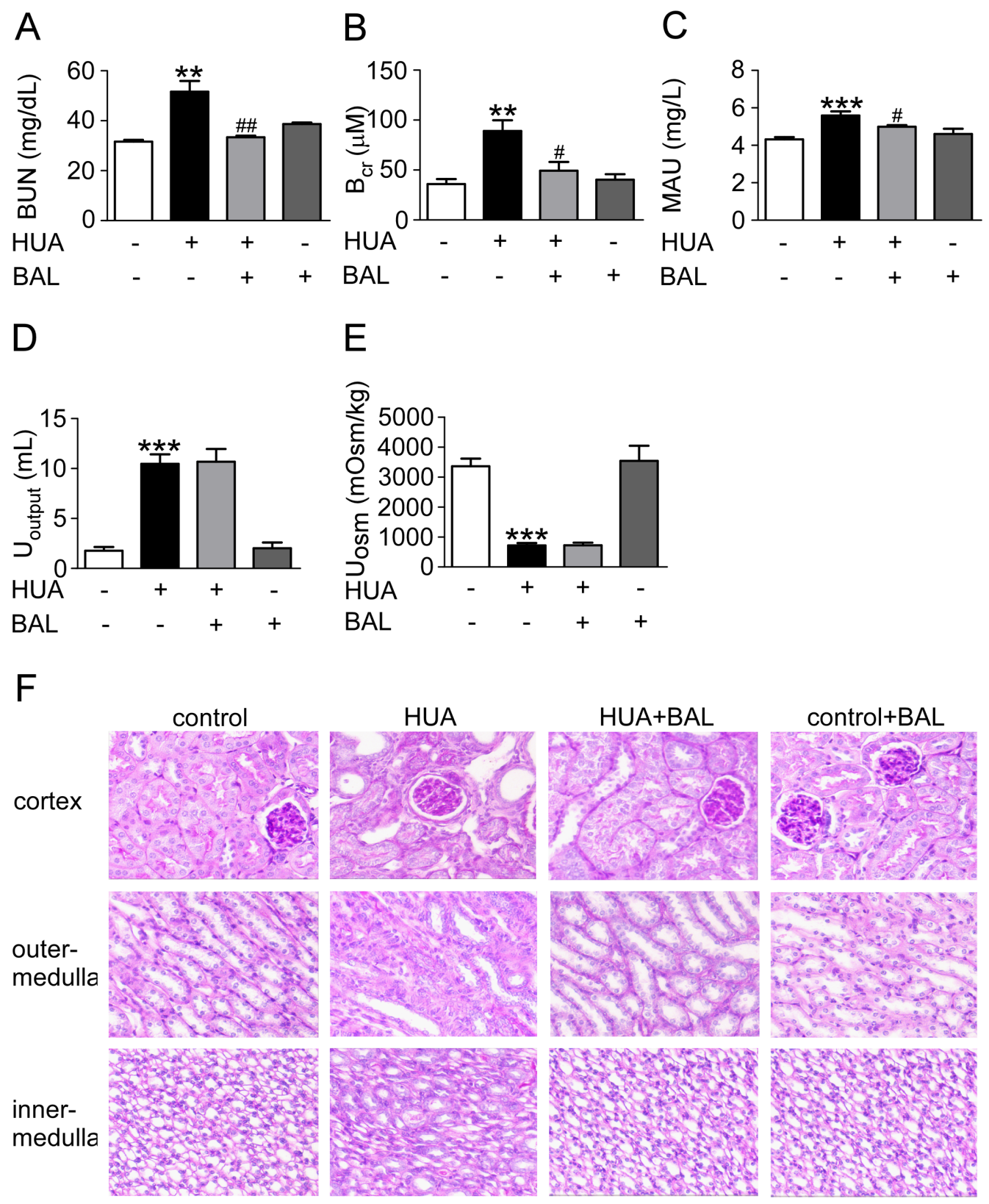

Figure 4: Baicalein improved renal function and kidney pathology in hyperuricemia mice. Blood, urine and kidney samples were collected for renal function tests and histological examination. (A) BUN. (B) Blood creatinine. (C) Urine microalbumin. (D) Urine output. (E) Urine osmolality. (F) Photomicrographs illustrated periodic acid-Schiff staining of the kidney tissues in control or hyperuricemia mice with or without baicalein treatment (magnification $200 \times$ ). Values were means $\pm \operatorname{SEM}(n=5-6)$. ${ }^{*} P<0.05$, $* * P<0.01$ and $* * * P<0.001$ vs. control group. ${ }^{\#} P<0.05,{ }^{\#} P<0.01$ vs. hyperuricemia group. 
not significantly changed after treatment with baicalein. We tested XOR expression in kidney also, but it was too low to be detected (data not shown). Thus, we concluded that baicalein conducted a uric acid-lowering effect through inhibiting XOR viability while not affecting its expression.

XOR acts as not only a producer of UA, but a major producer of reactive oxygen species (ROS) [32]. XOR releases $\mathrm{O}_{2}{ }^{-}$and $\mathrm{H}_{2} \mathrm{O}_{2}$, which could be converted to more toxic ROS, peroxynitrate $\left(\mathrm{ONOO}^{-}\right)$, hydroxyl anion $\left(\mathrm{OH}^{-}\right)$, and hypochorous acid $(\mathrm{HOCl})$. They all do harm to proteins, lipids, carbohydrates, DNA, RNA, subcellular organelles and cell systems [36]. XOR generates $\sim 25 \%$ $\mathrm{O}_{2}{ }^{-}$and $\sim 75 \% \mathrm{H}_{2} \mathrm{O}_{2}$ while the oxidative hydroxylation of xanthine to UA takes place [37]. So we detected $\mathrm{H}_{2} \mathrm{O}_{2}$ level and saw an increase in hyperuricemia mice and baicalein renormalized $\mathrm{H}_{2} \mathrm{O}_{2}$ concentration.

Oxidative stress is a disease mechanism common to a variety of disorders harming human health [39]. It can result in DNA damage, lipid peroxidation, protein modification, and other pathological effects in various chronic disorders, including neurodegenerative, cardiovascular and renal diseases, and cancer [40-43]. In organism, ROS levels are kept within a narrow range by enzymatic antioxidants, such as SOD, GPx, or nonenzymatic antioxidants, such as ascorbic acid, GSH [44]. Baicalein scavenged hydroxyl free radicals, $\mathrm{O}_{2}^{-}$, diphenylpicrylhydrazyl free radicals, and lipid peroxyl radicals [45]. Also, baicalein could inhibit $\mathrm{H}_{2} \mathrm{O}_{2}$ inducing RAW264.7 apoptosis, hypodiploid generation, DNA breakage, caspase-3 activation and cytochrome $\mathrm{C}$ release [46]. In our study, an increase of $\mathrm{H}_{2} \mathrm{O}_{2}$ level and a decrease of SOD, GPx and GSH activity were observed. Pretreatment of baicalein significantly reversed the situation. At the same time, hyperuricemia induced activation of NADPH oxidase and it was inhibited by baicalein. These results indicate that baicalein may block both of the XOR-dependent and NADPH oxidase-dependent production of ROS and enhance ROS elimination. This normalizes the imbalance between the oxidative and anti-oxidative status after hyperuricemia.

In addition to oxidative stress XOR brought about, we cannot ignore the damage caused by its final production-UA. Because of excessive UA production and decreased UA clearance, hyperuricemia is a common finding in CKD [8]. As baicalein inhibited XOR effectively

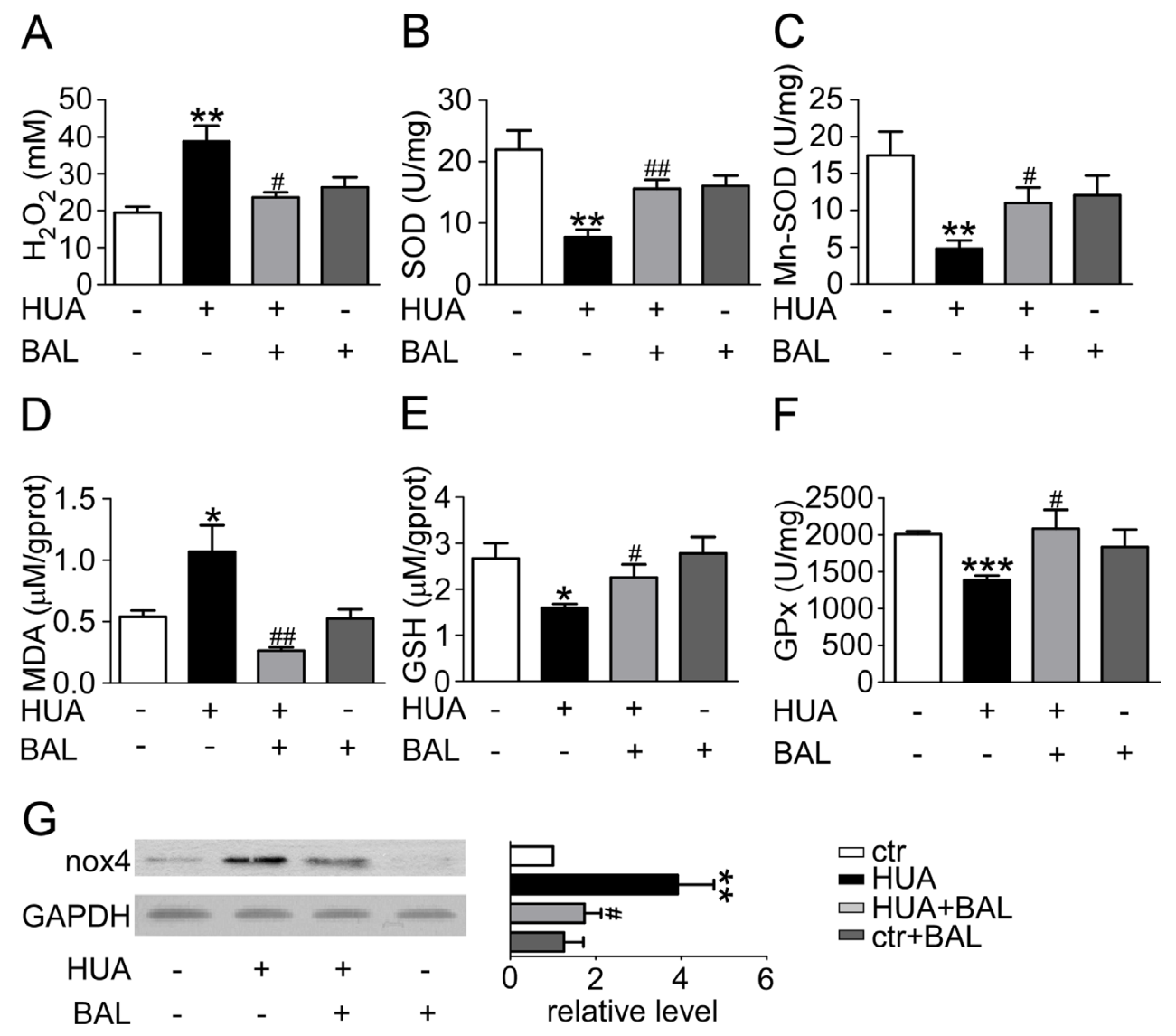

Figure 5: Baicalein prevented renal oxidative stress in hyperuricemia mice. Blood was collected to determine ROS level and kidney tissues were homogenized for evaluating the levels of different enzymes. (A) $\mathrm{H}_{2} \mathrm{O}_{2}$ level of mouse blood. (B) SOD activity in renal tissue. (C) Mn-SOD activity in renal tissue. (D) Malondialdehyde activity in renal tissue. (E) GSH level in renal tissue. (F) GPx activity in renal tissue. Means $\pm \operatorname{SEM}(n=5-6)$. ${ }^{*} P<0.05,{ }^{*} * P<0.01$ and $* * * P<0.001$ vs. control group. ${ }^{*} P<0.05,{ }^{\#} P<0.01$ and ${ }^{\# \#} P<0.001$ vs. hyperuricemia group. (G) Expression levels of Nox4 in kidney were determined by western blots (left) and quantifications (right), which were normalized with GAPDH. Means $\pm \operatorname{SEM}(n=4)$. 
(Figures 1,2) while had no influence on renal transporters (data not shown), baicalein possibly decreased UA level mainly through suppression of UA production. Although UA is a major antioxidant in human blood that may protect against aging and oxidative stress, it also causes or exacerbates inflammation, endothelial dysfunction, mesangial and fibroblast activation, and finally kidney fibrosis and progressive CKD [47-49]. Furthermore, UA may cause hypertension and metabolic syndrome, which are thought to be initiated by oxidative stress [50, 51]. In line with these studies, we found micro-albuminuria in hyperuricemia mice, which is a well-known early marker of CKD. And kidneys of hyperuricemia mice displayed severe morphologic lesions characterized by tubular dilation and interstitial collagen accumulation. So we focused on renal fibrosis and relevant pathways.

As MMPs greatly changed in our RNA-seq results (data not shown), we investigated their alterations in

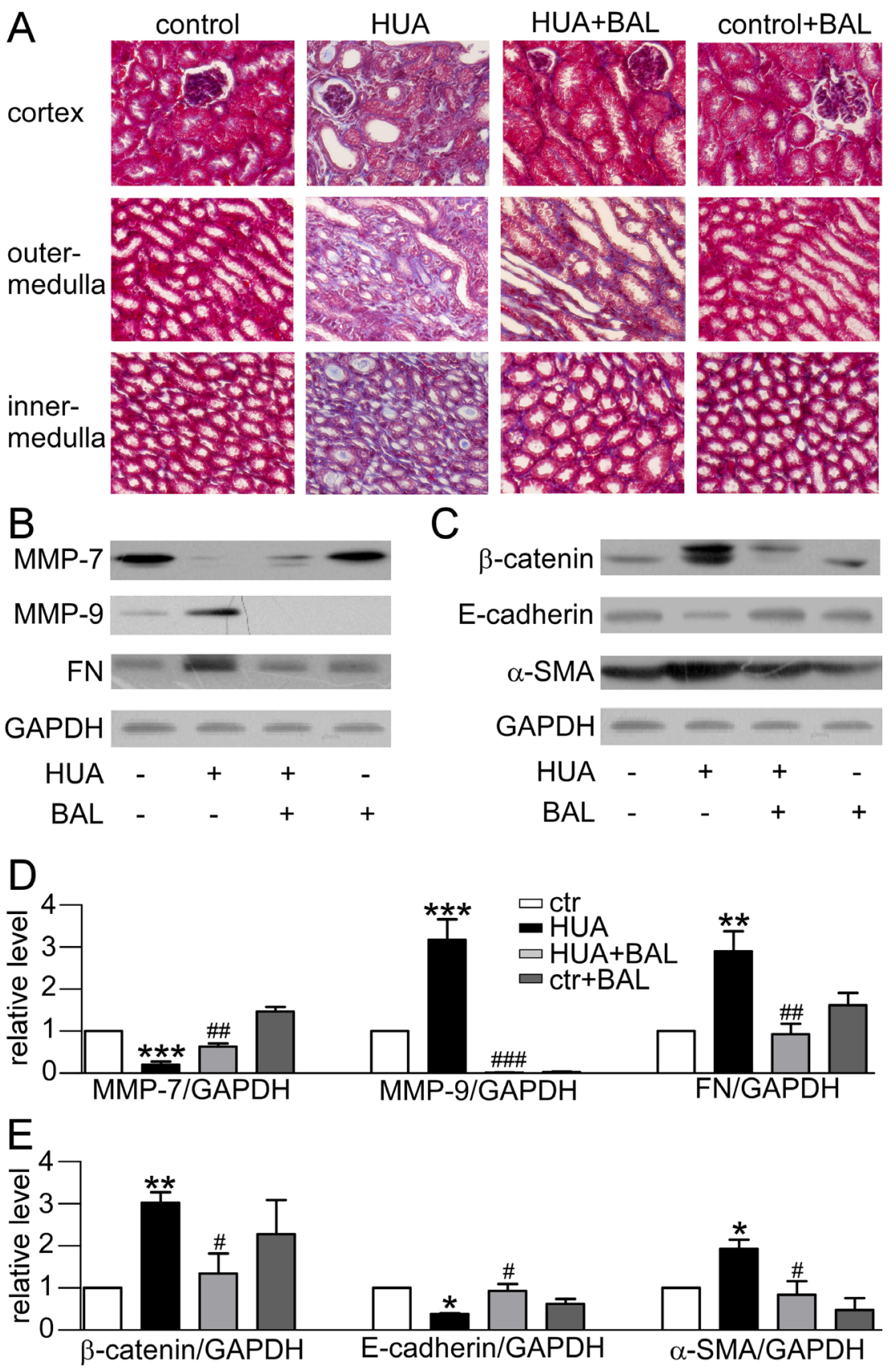

Figure 6: Effect of baicalein on fibrosis and EMT related proteins in mice. (A) Photomicrographs illustrated Masson trichrome staining of the kidney tissues in control or hyperuricemia mice with or without baicalein treatment (magnification 200×). (B) Western blots of fibrosis-related protein. (C) Western blots of EMT-related protein. (D) Quantification of fibrosis-related protein. (E) Quantification of EMT-related protein. The data were normalized by the intensity of GAPDH. Means $\pm \operatorname{SEM}(n=4) . * P<0.05, * * P<0.01$ and $* * * P<0.001$ vs. control group. ${ }^{\#} P<0.05,{ }^{\#} P<0.01$ and ${ }^{\# \#} P<0.001$ vs. hyperuricemia group. 
hyperuricemia. MMPs is a large family of zinc-dependent endopeptidases. They are collectively capable of proteolyzing all components of the extracellular matrix (ECM). MMPs in the pathogenesis of nephropathy is controversial and inconsistent [52]. They regulate fibrotic process either positively or negatively via targeting different sets of substrate proteins for degradation [53]. Interestingly, our data showed an elevated-MMP-9 level and a decreasedMMP-7 level in hyperuricemia mouse. This result is according with a wide variety studies recognizing MMP-9 as a biomarker of renal injury $[52,54]$. In terms of MMP-7, it may be more complicated. This trend is in line with elevated expression of fibronectin, which presents as the cleavage substrate of MMP-7. Apart from that, we blamed it to the overcapacity of ROS. The MMPs are synthesized as inactive zymogens maintained by a conserved cysteine residue. It forms Cys- $\mathrm{Zn}^{2+}$ coordination and prevents water molecules from catalysis [55]. However, the cysteine switch compasses a thiol residue. It can be altered by ROS and results in dissociation from the catalytic site and enzyme activation. So MMP-7 can be activated at lower concentrations of ROS. However, at a higher concentration of ROS, MMP-7 is inactivated by oxidizing specific amino acid residues. It affects the conformine of active site, possibly preventing overactivity $[52,56]$.

Reactivating and hyperactive $\beta$-catenin signaling is detrimental in glomeruli and tubules. Moreover, genetic and pharmacologic activation of $\beta$-catenin may cause proteinuria $[57,58]$. Accordingly, we have confirmed the activation of $\beta$-catenin and proteinuria in our hyperuricemia mice. MMP-7 and MMP-9 were also involved in $\beta$-catenin signal. They can promote degradation of E-cadherin, and the process leads to $\beta$-catenin release and activation $[59,60]$. At the same time, the stabilization and nuclear translocation of $\beta$-catenin stimulated the transcription of target genes in kidney, including fibronectin and MMP-7 itself as well [61]. As only MMP-9 level was elevated, we thought that maybe MMP-9 played a more important role in this pathway. Based on previous reports, the decreased MMP-7 level might be concerned with the overproduction of ROS (Figure 7).

Meanwhile, we found an increased expression of $\alpha$-SMA and a decreased level of E-cadherin, which indicated EMT process. EMT is a major source of fibroblasts in tissue fibrosis and MMPs are part of its regulation [52]. Both MMP-7 and MMP-9 could induce EMT in renal tubular cell cultures through cleavage of the epithelial cell marker E-cadherin [53, 62]. In our work, baicalein alleviated fibrosis and EMT through above pathways effectively. Therefore, our studies highlight that baicalein can alleviate fibrosis and EMT possibly through MMP-9/ $\beta$-catenin signaling and MMP-7 in hyperuricemic nephropathy.

In summary, we are the first to demonstrate that baicalein can attenuate development of hyperuricemiainduced nephropathy. This beneficial effect may be attributed to XOR inhibition, NADPH oxidase-dependent

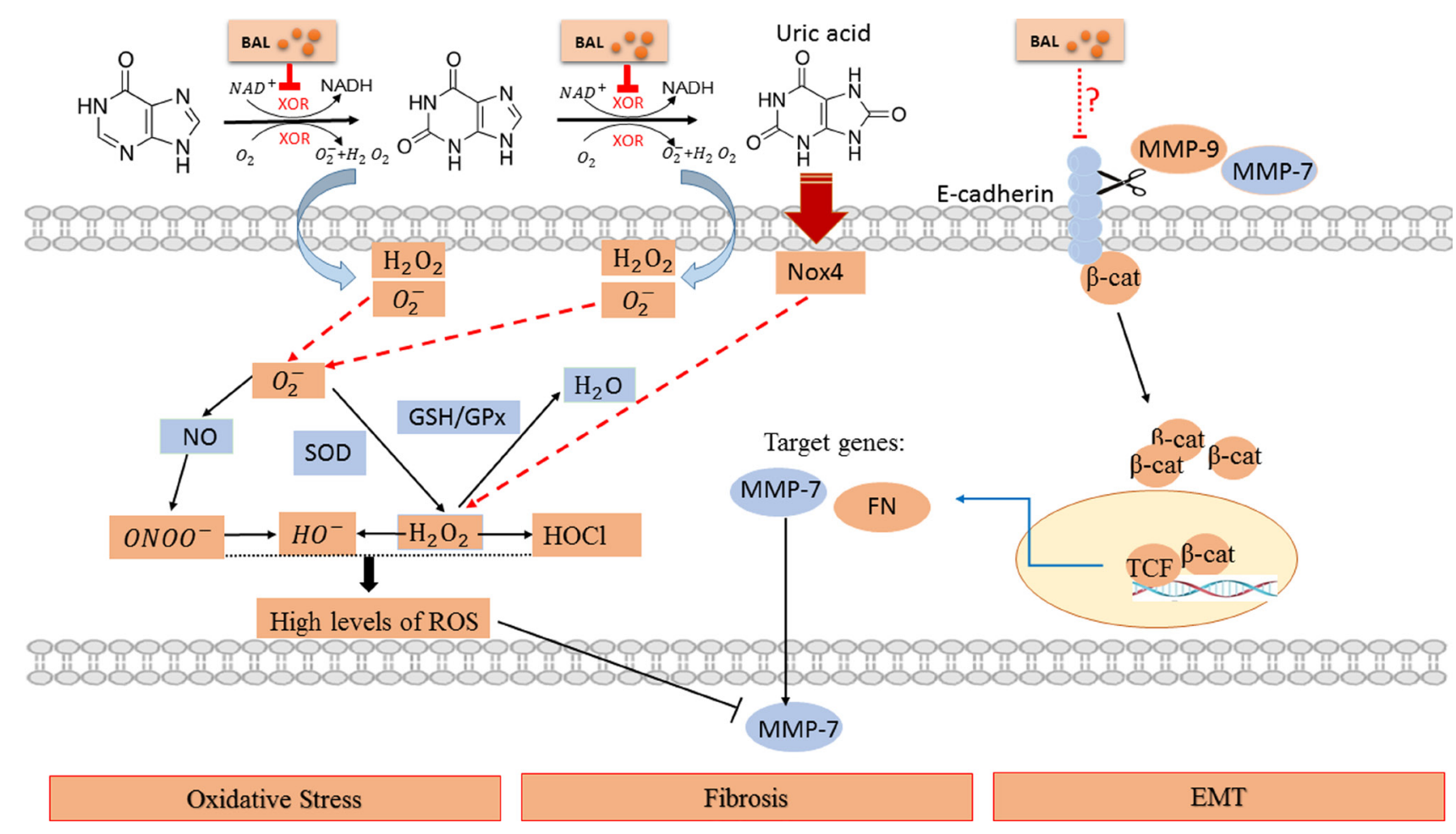

Figure 7: Schematic diagram of the potential pathway in hyperuricemia and interfered by baicalein. Baicalein significantly suppressed the viability of XOR and remarkably prevented renal dysfunction, ameliorated kidney fibrosis, alleviated EMT and oxidative stress in hyperuricemia mice. Please see the text for more details. 
and XOR-dependent ROS elimination, EMT moderation and fibrosis alleviation. Thus, baicalein may be developed as a candidate drug for hyperuricemia and hyperuricemia related other diseases.

\section{MATERIALS AND METHODS}

\section{Ethics statement}

All procedures in this study were carried out in strict accordance with the recommendations of the Guide for the Care and Use of Laboratory Animals of China Association for Laboratory Animal Science. All animal care protocols were approved by the Animal Care Committee of Peking University Health Science Center. All sacrifices were performed under pentobarbitone anesthesia, and every effort was made to minimize animal suffering.

\section{Baicalein}

Baicalein is kindly provided by Topscience BioPharm Technology. The compound's average molecular weight is approximately 270, as determined by highperformance steric exclusion chromatography analysis. In our experiments, baicalein was dissolved in sodium carboxymethyl cellulose (CMC) for animal treatment.

\section{Molecular docking}

To simulate the binding modes of baicalein with two receptors: rat XOR (pdb entry: $1 \mathrm{WYG}$ ) and human XOR (pdb entry: 2E1Q), molecular docking studies were performed via inserting baicalein into corresponding binding sites in crystal structures. The structure of baicalein was built using a 2D/3D editor sketcher in Catalyst software and the energy has been minimized. Parameters of docking were set in default. 20 conformations per calculation were generated via molecular docking and the best complex was chosen by docking total score.

\section{SPR biosensor analysis}

The binding affinity of baicalein to $\mathrm{XOR}$ in vitro was assayed using the SPR-based Biacore 3000 instrument (Biacore AB, Uppsala, Sweden). XOR protein (molecular mass, $160 \mathrm{kDa}$ ) was purchased from Sigma-Aldrich. The XOR protein was immobilized on a CM5 sensor chip according to the standard procedures. The data were collected at a constant HBS-EP flow rate of $30 \mu \mathrm{l} / \mathrm{min}$ at $25^{\circ} \mathrm{C}$. Baicalein was diluted into the running buffer to create a series of concentrations from $12.5 \mu \mathrm{M}$ down to $0.78125 \mu \mathrm{M}$. The samples were injected into the channels at a flow rate of $30 \mu \mathrm{l} / \mathrm{min}$ and the binding responses were continuously recorded in RU. The association $\left(\mathrm{K}_{\mathrm{on}}\right)$ and dissociation $\left(\mathrm{K}_{\text {off }}\right)$ rate constants and the equilibrium dissociation constant $\left(\mathrm{K}_{\mathrm{D}}=\mathrm{K}_{\text {off }}\right)$ were calculated using
BIA evaluation software version 3.1 (Biacore) with 1:1 Langmuir binding fitting model applied.

\section{Hyperuricemia mouse model}

Male ICR mice ( 8 weeks -10 weeks old) weighing 20 g-22 g were purchased from the Animal Center of Peking University Health Science Center. The mice were acclimated to this environment for $7 \mathrm{~d}$ before experiments. The mice were divided randomly into four groups: the sham-operated group; the sham-operated baicalein (50 mg/kg)-treated group; the hyperuricemia group; and the hyperuricemia-baicalein $(50 \mathrm{mg} / \mathrm{kg})$-treated group. The hyperuricemia mouse model was established by peritoneal injection of a mixture of hypoxanthine $(0.3 \mathrm{~g} / \mathrm{kg})$ and potassium oxonate $(0.3 \mathrm{~g} / \mathrm{kg})$ daily consistently for 2 weeks. In order to analyze the prevention efficacy of baicalein, it was orally injected for 21 days until sacrifice, including 7 days pretreatment. Animals treated with CMC alone were used as controls. After that, the animals were euthanized and the kidneys and livers were collected for protein analysis and histologic examination. Blood was also taken for the measurement of serum uric acid, BUN, creatinine, and other biochemistry indices. Twenty-fourhour urine samples were collected in metabolic cages at the last day for determination of urinary levels of protein.

\section{Assessment of UA, renal function, and other biochemistry indices}

Serum UA, creatinine, BUN and urine UA were determined by commercial kits (NJC Bio) according to the manufacturer's instructions. Urinary osmolality was measured using freezing point depression (Microosmometer, FISKER ASSOCIATES Q23). Microal buminuria was tested with ELISA kit (Wuhan Xinqidi Biological Technology Co., Ltd.).

\section{Assessment of serum activity of XOR}

Serum activity of XOR was examined according to the protocol provided by the manufacture (NJJC Bio).

\section{Western blot analysis}

Total protein was extracted using RIPA lysis buffer, and equal amounts of proteins were subjected to $8 \%$ SDS-PAGE and then transferred to polyvinylidene difluoride membranes (Millipore Corp., MA, U.S.A). The membranes were blocked and then incubated in primary antibodies against GAPDH (ABclonal Technology, China), fibronectin, MMP-7, MMP-9, Nox4 (ABcam), $\beta$-catenin (Epitomics), E-cadherin (Bioworld, China), $\alpha$-SMA (Huaxing Bio., China) overnight at $4^{\circ} \mathrm{C}$ with gentle agitation, followed by incubation in the goat antirabbit IgG or goat anti-mouse IgG (Santa Cruz) labeled 
secondary antibody for $45 \mathrm{~min}$ at room temperature. Three 10-min washes in TBST were performed after secondary antibody labeling. The blots were developed with an ECL plus kit (Amersham Biosciences). The images were scanned with an Epson scanning system, and the data were analyzed with Quantity-one software. The data are expressed as the values relative to the sham or control value. When probing for multiple targets, stripping and re-probing a single membrane was needed. The membrane was incubated in stripping solution (Pplygen) for $20 \mathrm{~min}$ at room temperature with gentle agitation, followed by a 5 min wash in TBST. The membrane was blocked and then incubated in another primary antibody. Then the procedures above were conducted.

\section{Masson trichrome staining and periodic acid- schiff staining}

Formalin-fixed kidneys were embedded in paraffin and prepared in 6 - $\mu \mathrm{m}$ - thick sections. Masson trichrome staining was performed according to the protocol provided by the manufacture (Sigma-Aldrich) to evaluate renal fibrosis, and Periodic acid-Schiff was conducted to test general histology.

\section{Statistical analyses}

All the experiments were performed at least three times. All results are represented as the mean $\pm \mathrm{SEM}$. Data involving only two groups was analyzed by $t$-test. A $p$-value of $<0.05$ was considered to be statistically significant difference for all tests.

\section{Abbreviations}

uric acid)UA; chronic kidney disease (CKD); xanthine oxidoreductase (XOR); Surface Plasmon Resonance (SPR); epithelial-mesenchymal transition (EMT); ST-segment elevation myocardial infarction (STEMI); superoxide dismutase (SOD); reduced glutathione (GSH); glutathione peroxidase (GPx); NADPH oxidase 4 (Nox4); matrix metalloproteinases (MMPs); $\alpha$-smooth muscle actin ( $\alpha$-SMA); reactive oxygen species (ROS); extracellular matrix (ECM); carboxymethyl cellulose $(\mathrm{CMC})$; hydroxyl anion $\left(\mathrm{OH}^{-}\right)$; hypochorous acid $(\mathrm{HOCl})$.

\section{Authors' contributions}

H.Z. is correspondence author. H.Z. and B.Y. conceived the study. H.Z., B.Y and X.M analyzed and interpreted the data. X.M., Z.M., X.L., D.Z., M.L., Y.J and J.W. performed the experiments. X.M., H.Z., and B.Y. wrote the manuscript. Final approval was given by all authors.

\section{CONFLICTS OF INTEREST}

The authors declare that there are no conflicts of interest.

\section{FUNDING}

This work was supported by National Natural Science Foundation of China grants 81370783, 81500535 and 81601103 and Leading Academic Discipline Project of Beijing Education Bureau, BMU20110254.

\section{REFERENCES}

1. Tasic V, Janchevska A, Emini N, Sahpazova E, Gucev Z, Polenakovic M. Chronic kidney disease-pediatric risk factors. Pril (Makedon Akad Nauk Umet Odd Med Nauki). 2016; 37:9-13.

2. Acevedo A, Benavides J, Chowdhury M, Lopez M, Pena L, Montenegro A, Lievano M, Lombo B. Hyperuricemia and Cardiovascular Disease in Patients with Hypertension. Conn Med. 2016; 80:85-90.

3. Jalal DI. Hyperuricemia, the kidneys, and the spectrum of associated diseases: a narrative review. Curr Med Res Opin. 2016; 32:1863-1869.

4. Kawada T. Cross-sectional and longitudinal study on the association between serum uric acid and metabolic syndrome. Clin Chim Acta. 2016; 455:201.

5. Shao X, Lu W, Gao F, Li D, Hu J, Li Y, Zuo Z, Jie H, Zhao Y, Cen X. Uric Acid Induces Cognitive Dysfunction through Hippocampal Inflammation in Rodents and Humans. J Neurosci. 2016; 36:10990-11005.

6. Liu CW, Liao PC, Chen KC, Chiu YW, Liu YH, Ke SR, $\mathrm{Wu}$ YW. Relationship of serum uric acid and Killip class on mortality after acute ST-segment elevation myocardial infarction and primary percutaneous coronary intervention. Int J Cardiol. 2016; 226:26-33.

7. Yasu T, Kobayashi S, Horii M, Kurokawa Y. The efficacy of febuxostat $10 \mathrm{mg}$ for the prevention of hyperuricemia associated with tumor lysis syndrome (TLS) in Japanese patients with non-Hodgkin's lymphoma. Int J Clin Pharmacol Ther. 2016; 54:1009-1012.

8. Kim IY, Lee DW, Lee SB. The role of uric acid in kidney fibrosis: experimental evidences for the causal relationship. 2014; 2014:638732.

9. Nashar K, Fried LF. Hyperuricemia and the progression of chronic kidney disease: is uric acid a marker or an independent risk factor? Adv Chronic Kidney Dis. 2012; 19:386-391.

10. Feig DI. Uric acid: a novel mediator and marker of risk in chronic kidney disease? Curr Opin Nephrol Hypertens. 2009; 18:526-530. 
11. Dousdampanis P, Trigka K, Musso CG, Fourtounas C. Hyperuricemia and chronic kidney disease: an enigma yet to be solved. Ren Fail. 2014; 36:1351-1359.

12. Stamp L, Dalbeth N. Urate-lowering therapy for asymptomatic hyperuricaemia: A need for caution. Semin Arthritis Rheum. 2016.

13. Levy G, Cheetham TC. Is It Time to Start Treating Asymptomatic Hyperuricemia? Am J Kidney Dis. 2015; 66:933-935.

14. Wang H, Wei Y, Kong X, Xu D. Effects of urate-lowering therapy in hyperuricemia on slowing the progression of renal function: a meta-analysis. J Ren Nutr. 2013; 23:389-396.

15. Okamoto K, Matsumoto K, Hille R, Eger BT, Pai EF, Nishino T. The crystal structure of xanthine oxidoreductase during catalysis: implications for reaction mechanism and enzyme inhibition. Proc Natl Acad Sci U S A. 2004; 101:7931-7936.

16. Okamoto K, Eger BT, Nishino T, Kondo S, Pai EF, Nishino T. An extremely potent inhibitor of xanthine oxidoreductase. Crystal structure of the enzyme-inhibitor complex and mechanism of inhibition. J Biol Chem. 2003; 278:1848-1855.

17. Dalbeth N, Merriman TR, Stamp LK. Gout. Lancet. 2016; 388:2039-2052.

18. Khanna D, Fitzgerald JD, Khanna PP, Bae S, Singh MK, Neogi T, Pillinger MH, Merill J, Lee S, Prakash S, Kaldas M, Gogia M, Perez-Ruiz F, et al. 2012 American College of Rheumatology guidelines for management of gout. Part 1: systematic nonpharmacologic and pharmacologic therapeutic approaches to hyperuricemia. Arthritis Care Res (Hoboken). 2012; 64:1431-1446.

19. Lin J, Chen S, Li S, Lu M, Li Y. Efficacy and Safety of Chinese Medicinal Herbs for the Treatment of Hyperuricemia: A Systematic Review and MetaAnalysis. Evid Based Complement Alternat Med. 2016; 2016:2146204.

20. Tsai KL, Hung CH, Chan SH, Shih JY, Cheng YH, Tsai YJ, Lin HC, Chu PM. Baicalein protects against oxLDL-caused oxidative stress and inflammation by modulation of AMPKalpha. Oncotarget. 2016; 7:72458-72468. doi: 10.18632/ oncotarget.12788.

21. Gaire BP, Moon SK, Kim H. Scutellaria baicalensis in stroke management: nature's blessing in traditional Eastern medicine. Chin J Integr Med. 2014; 20:712-720.

22. Li J, Ma J, Wang KS, Mi C, Wang Z, Piao LX, Xu GH, Li X, Lee JJ, Jin X. Baicalein inhibits TNF-alpha-induced NFkappaB activation and expression of NF-kappaB-regulated target gene products. Oncol Rep. 2016; 36:2771-2776.

23. Liu H, Dong Y, Gao Y, Du Z, Wang Y, Cheng P, Chen A, Huang H. The Fascinating Effects of Baicalein on Cancer: A Review. Int J Mol Sci. 2016; 17.

24. Wang W, Zhou PH, Xu CG, Zhou XJ, Hu W, Zhang J. Baicalein attenuates renal fibrosis by inhibiting inflammation via down-regulating NF-kappaB and MAPK signal pathways. J Mol Histol. 2015; 46:283-290.

25. Wang W, Zhou PH, Xu CG, Zhou XJ, Hu W, Zhang J. Baicalein ameliorates renal interstitial fibrosis by inducing myofibroblast apoptosis in vivo and in vitro. BJU Int. 2016; 118:145-152.

26. Zhao F, Fu L, Yang W, Dong Y, Yang J, Sun S, Hou Y. Cardioprotective effects of baicalein on heart failure via modulation of $\mathrm{Ca}(2+)$ handling proteins in vivo and in vitro. Life Sci. 2016; 145:213-223.

27. Chen M, Lai L, Li X, Zhang X, He X, Liu W, Li R, Ke X, Fu C, Huang Z, Duan C. Baicalein Attenuates Neurological Deficits and Preserves Blood-Brain Barrier Integrity in a Rat Model of Intracerebral Hemorrhage. Neurochem Res. 2016; 41:3095-3102.

28. Ribeiro AR, do Nascimento Valenca JD, da Silva Santos J, Boeing T, da Silva LM, de Andrade SF, AlbuquerqueJunior RL, Thomazzi SM. The effects of baicalein on gastric mucosal ulcerations in mice: Protective pathways and antisecretory mechanisms. Chem Biol Interact. 2016; 260:33-41.

29. Isaka Y, Takabatake Y, Takahashi A, Saitoh T, Yoshimori T. Hyperuricemia-induced inflammasome and kidney diseases. Nephrol Dial Transplant. 2016; 31:890-896.

30. Nishino T, Okamoto K. Mechanistic insights into xanthine oxidoreductase from development studies of candidate drugs to treat hyperuricemia and gout. J Biol Inorg Chem. 2015; 20:195-207.

31. Lin S, Zhang G, Liao Y, Pan J. Inhibition of chrysin on xanthine oxidase activity and its inhibition mechanism. Int J Biol Macromol. 2015; 81:274-282.

32. Chen C, Lu JM, Yao Q. Hyperuricemia-Related Diseases and Xanthine Oxidoreductase (XOR) Inhibitors: An Overview. Med Sci Monit. 2016; 22:2501-2512.

33. Rashidi MR, Nazemiyeh H. Inhibitory effects of flavonoids on molybdenum hydroxylases activity. Expert Opin Drug Metab Toxicol. 2010; 6:133-152.

34. Lin S, Zhang G, Liao Y, Pan J, Gong D. Dietary Flavonoids as Xanthine Oxidase Inhibitors: Structure-Affinity and Structure-Activity Relationships. J Agric Food Chem. 2015; 63:7784-7794.

35. Battelli MG, Bolognesi A, Polito L. Pathophysiology of circulating xanthine oxidoreductase: new emerging roles for a multi-tasking enzyme. Biochim Biophys Acta. 2014; 1842:1502-1517.

36. Chen AY, Lu JM, Yao Q, Chen C. Entacapone is an Antioxidant More Potent than Vitamin C and Vitamin E for Scavenging of Hypochlorous Acid and Peroxynitrite, and the Inhibition of Oxidative Stress-Induced Cell Death. Med Sci Monit. 2016; 22:687-696.

37. Kelley EE. A new paradigm for XOR-catalyzed reactive species generation in the endothelium. Pharmacol Rep. 2015; 67:669-674.

38. Battelli MG, Polito L. Xanthine Oxidoreductase-Derived Reactive Species: Physiological and Pathological Effects. Oxid Med Cell Longev. 2016; 2016:3527579. 
39. Dao VT, Casas AI, Maghzal GJ, Seredenina T, Kaludercic N, Robledinos-Anton N, Di Lisa F, Stocker R, Ghezzi P, Jaquet V, Cuadrado A, Schmidt HH. Pharmacology and Clinical Drug Candidates in Redox Medicine. Antioxid Redox Signal. 2015; 23:1113-1129.

40. Smith JA, Park S, Krause JS, Banik NL. Oxidative stress, DNA damage, and the telomeric complex as therapeutic targets in acute neurodegeneration. Neurochem Int. 2013; 62:764-775.

41. Loperena R, Harrison DG. Oxidative Stress and Hypertensive Diseases. Med Clin North Am. 2017; 101:169-193.

42. Bhatti JS, Bhatti GK, Reddy PH. Mitochondrial dysfunction and oxidative stress in metabolic disorders - A step towards mitochondria based therapeutic strategies. Biochim Biophys Acta. 2016; 1863:1066-1077.

43. Kim J, Kim J, Bae JS. ROS homeostasis and metabolism: a critical liaison for cancer therapy. Exp Mol Med. 2016; 48:e269.

44. Koskenkorva-Frank TS, Weiss G, Koppenol WH, Burckhardt S. The complex interplay of iron metabolism, reactive oxygen species, and reactive nitrogen species: insights into the potential of various iron therapies to induce oxidative and nitrosative stress. Free Radic Biol Med. 2013; 65:1174-1194.

45. Gao Z, Huang K, Yang X, Xu H. Free radical scavenging and antioxidant activities of flavonoids extracted from the radix of Scutellaria baicalensis Georgi. Biochim Biophys Acta. 1999; 1472:643-650.

46. Lin HY, Shen SC, Lin CW, Yang LY, Chen YC. Baicalein inhibition of hydrogen peroxide-induced apoptosis via ROS-dependent heme oxygenase 1 gene expression. Biochim Biophys Acta. 2007; 1773:1073-1086.

47. Luo C, Lian X, Hong L, Zou J, Li Z, Zhu Y, Huang T, Zhang Y, Hu Y, Yuan H, Wen T, Zhuang W, Cai B, et al. High Uric Acid Activates the ROS-AMPK Pathway, Impairs CD68 Expression and Inhibits OxLDL-Induced Foam-Cell Formation in a Human Monocytic Cell Line, THP-1. Cell Physiol Biochem. 2016; 40:538-548.

48. Wang M, Zhao J, Zhang N, Chen J. Astilbin improves potassium oxonate-induced hyperuricemia and kidney injury through regulating oxidative stress and inflammation response in mice. Biomed Pharmacother. 2016; 83:975-988.

49. Liu N, Wang L, Yang T, Xiong C, Xu L, Shi Y, Bao W, Chin YE, Cheng SB, Yan H, Qiu A, Zhuang S. EGF Receptor Inhibition Alleviates Hyperuricemic Nephropathy. J Am Soc Nephrol. 2015; 26:2716-2729.

50. Canepa M, Viazzi F, Strait JB, Ameri P, Pontremoli R, Brunelli C, Studenski S, Ferrucci L, Lakatta EG, AlGhatrif M. Longitudinal Association Between Serum Uric Acid and Arterial Stiffness: Results From the Baltimore Longitudinal Study of Aging. Hypertension. 2016.

51. Prasad M, Matteson EL, Herrmann J, Gulati R, Rihal CS, Lerman LO, Lerman A. Uric Acid Is Associated With
Inflammation, Coronary Microvascular Dysfunction, and Adverse Outcomes in Postmenopausal Women. Hypertension. 2016; 69:228-235.

52. Tan RJ, Liu Y. Matrix metalloproteinases in kidney homeostasis and diseases. Am J Physiol Renal Physiol. 2012; 302:F1351-1361.

53. Zhou D, Tian Y, Sun L, Zhou L, Xiao L, Tan RJ, Tian J, Fu H, Hou FF, Liu Y. Matrix Metalloproteinase-7 Is a Urinary Biomarker and Pathogenic Mediator of Kidney Fibrosis. J Am Soc Nephrol. 2016; 28:598-611.

54. Hopps E, Caimi G. Matrix metalloproteases as a pharmacological target in cardiovascular diseases. Eur Rev Med Pharmacol Sci. 2015; 19:2583-2589.

55. Nagase H, Visse R, Murphy G. Structure and function of matrix metalloproteinases and TIMPs. Cardiovasc Res. 2006; 69:562-573.

56. Fu X, Kao JL, Bergt C, Kassim SY, Huq NP, d'Avignon A, Parks WC, Mecham RP, Heinecke JW. Oxidative cross-linking of tryptophan to glycine restrains matrix metalloproteinase activity: specific structural motifs control protein oxidation. J Biol Chem. 2004; 279:6209-6212.

57. Kato H, Gruenwald A, Suh JH, Miner JH, BarisoniThomas L, Taketo MM, Faul C, Millar SE, Holzman LB, Susztak K. Wnt/beta-catenin pathway in podocytes integrates cell adhesion, differentiation, and survival. J Biol Chem. 2011; 286:26003-26015.

58. Dai C, Stolz DB, Kiss LP, Monga SP, Holzman LB, Liu Y. Wnt/beta-catenin signaling promotes podocyte dysfunction and albuminuria. J Am Soc Nephrol. 2009; 20:1997-2008.

59. Kim H, He Y, Yang I, Zeng Y, Kim Y, Seo YW, Murnane MJ, Jung C, Lee JH, Min JJ, Kwon DD, Kim KK, Lu Q, et al. delta-Catenin promotes E-cadherin processing and activates beta-catenin-mediated signaling: implications on human prostate cancer progression. Biochim Biophys Acta. 2012; 1822:509-521.

60. Padwal M, Siddique I, Wu L, Tang K, Boivin F, Liu L, Robertson J, Bridgewater D, West-Mays J, Gangji A, Brimble KS, Margetts PJ. Matrix metalloproteinase 9 is associated with peritoneal membrane solute transport and induces angiogenesis through beta-catenin signaling. Nephrol Dial Transplant. 2016; 32:50-61.

61. He W, Tan RJ, Li Y, Wang D, Nie J, Hou FF, Liu Y. Matrix metalloproteinase-7 as a surrogate marker predicts renal Wnt/beta-catenin activity in CKD. J Am Soc Nephrol. 2012; 23:294-304.

62. Zheng G, Lyons JG, Tan TK, Wang Y, Hsu TT, Min D, Succar L, Rangan GK, Hu M, Henderson BR, Alexander SI, Harris DC. Disruption of E-cadherin by matrix metalloproteinase directly mediates epithelial-mesenchymal transition downstream of transforming growth factorbeta1 in renal tubular epithelial cells. Am J Pathol. 2009; 175:580-591. 